\title{
5 Spectrum Arguments, Indeterminacy, and Value Superiority
}

\author{
Henrik Andersson
}

\section{Introduction $^{1}$}

The assumption that the value comparative "better than" is transitive is central to most normative reasoning. ${ }^{2}$ If it is not a transitive relation then there may be alternatives, such as $A, B$, and $C$ for which $A$ is better than $B, B$ is better than $C$, and yet $C$ is better than $A$. This possibility would make it impossible to pick a best alternative since it allows for a cycle. However, in his book Rethinking the Good: Moral Ideals and the Nature of Practical Reasoning, Larry Temkin (2012) forcefully questions the assumption that "better than" is a transitive relation. ${ }^{3}$ Roughly, his explanation as to why our value relations might not be transitive is that, depending on what I compare a specific object with, different considerations will be more or less weighty. Or, as Temkin (2012) puts it, the assessment of relative goodness is sometimes "essentially comparative." When comparing $A$ with $B$, some specific consideration is central, and $A$ is better than $B$ with respect to this consideration. The same consideration may be central when comparing $B$ with $C$, and $B$ is better than $C$ with respect to this consideration. However, when comparing $C$ with $A$, some other consideration may be more central, making $C$ better than $A$. This can explain the potential cyclicity.

Temkin's arguments in favor of this possibility are very detailed and sophisticated; it is impossible to do justice to all of them here. However, his spectrum arguments are central to his claim. Temkin uses spectrum arguments to argue that the belief that the "better than" relation is transitive is incompatible with other more intuitive beliefs, so we should conclude that "better than" is not transitive. While there are several possible formulations of spectrum arguments, I will focus on the formulation of the argument that Temkin deems "the simplest, most powerful counterexample to the transitivity of the betterness relation" (Temkin 2012: 135). ${ }^{4}$ If it can be shown that Temkin's most convincing formulation of the argument fails, then this casts doubt on the endeavor in general.

I will argue that Temkin's argument does not threaten the transitivity of betterness. First, I will provide an informal account of why his 


\section{Henrik Andersson}

argument fails. Roughly, I will argue that if we acknowledge that there can be a zone of vagueness in Temkin's spectrum argument, then we need not accept his conclusion. Second, I will show how the formal features of our value relations provide a neat explanation as to why we ought not to reject the transitivity of the betterness relation and that we instead should reject a belief expressed by Temkin about evaluative trade-offs. It is important to note, in the context of this book, that my rebuttal of Temkin's argument is conservative in that it does not refer to nonconventional value relations such as parity, imprecision, or rough equality. As such, my objection could be understood to be part of the larger effort to provide an account of the normative landscape without referring to non-conventional value relations; the "at least as good" relation and vagueness suffice. ${ }^{5}$

\section{The Spectrum Argument}

Temkin's preferred formulation of the spectrum argument starts by noting that the following views seem intuitively appealing:

View One: For any unpleasant or "negative" experience, no matter what the intensity and duration of that experience, it would be better to have the experience than one that was only a "little" less intense but twice (or three or five times) as long.

View Two: There is, or could be, a spectrum of unpleasant or "negative" experiences ranging in intensity, for example, from extreme forms of torture to the mild discomfort of a mosquito bite, such that one could move from the harsh end of the spectrum to the mild end in a finite series of steps, where each step would involve the transformation from one negative experience to another that was only a "little" less intense than the previous one.

View Three: The mild discomfort of a mosquito bite would be better than two years of excruciating torture, no matter how long one lived and no matter how long the discomfort of a mosquito bite persisted.

View Four: "All-things-considered better than" is a transitive relation. So, for any three outcomes, A, B, and C, which involve unpleasant experiences of varying intensities and durations, if, all things considered, A is better than B and B is better than C, then A is better than C.

(Temkin 2012: 135)

Now consider a series of lives from $P_{1}$ to $P_{n}$. All these lives are very lengthy and all include 15 mosquito bites per month. $P_{1}$ and $P_{2}$ are similar in all relevant respects, except that $P_{1}$ includes two years of excruciating torture, while $P_{2}$ includes four years of almost as intense torture. $P_{3}$ 
stands in the same relation to $P_{2}$ as $P_{2}$ does to $P_{1}$, i.e., $P_{3}$ includes eight years of almost as intense torture as does $P_{2}$. We would say, in accordance with View One, that $P_{1}$ is better than $P_{2}$, and that $P_{2}$ is better than $P_{3}$, and so on. However, in accordance with View Two, we will eventually reach $P_{n}$, which is such that it does not include excruciating torture, but very minor discomfort for a very long time. It may very well be that this minor discomfort is just one extra mosquito bite per month. Given View Four, $P_{1}$ is better than $P_{n}$, but according to View Three, $P_{n}$ is better than $P_{1}$. It has thus been shown that Views One, Two, Three, and Four are incompatible. Something has to go, but all four views seem correct. Moreover, rejecting any of the four views seems to lead to both practical and theoretical problems. ${ }^{6}$ Temkin argues that it is View Four that must be rejected, as rejecting any of the other views would come at too high a price.

\section{The Spectrum Argument and Indeterminacy}

There are, of course, ways in which one can object to spectrum arguments of this type, and Temkin discusses several such objections. There is, however, one type of objection I wish to discuss further. This objection is that there must be a point in the spectrum where View One does not apply between two adjacent outcomes, breaking the chain of "better than" relations. ${ }^{7}$ One explanation as to why that might be the case is that differences in degree may give rise to a difference in kind, and since there clearly is a difference in degree in the spectrum argument, there could also be a difference in kind. That is, not only do the pains differ in intensity, but the experiences also differ in a more fundamental manner that cannot be fully accounted for in terms of intensity - i.e., they have different phenomenological characteristics. This difference in kind becomes clear when one considers the difference between excruciating torture and a mosquito bite. Such a difference is not merely a difference in degree but also a difference in kind; they belong to two different categories of experiences. If there is a difference in kind between experiences at the opposite ends of the spectrum, then we should expect this difference in kind to arise somewhere in the spectrum. That is, a difference in kind can also, albeit less obviously, be found between two adjacent experiences in the spectrum. This means that even though two adjacent experiences are similar, there could be a difference in kind between them such that when we compare them the factors that are relevant and significant for comparing other adjacent experiences in the spectrum no longer apply (Temkin 2012: 266). The simple trade-off expressed in View One does not hold for these experiences since the pains are of a sufficiently different kind.

Temkin admits that there are such differences in kind in the spectrum; the reason that View Three applies to experiences at the opposite ends 


\section{Henrik Andersson}

of the spectrum is that there is a difference in kind between these experiences, excruciating torture differs in kind from a mosquito bite. Temkin even takes this to explain why transitivity does not hold. Due to the difference in kind between experiences at the opposite ends of the spectrum, factors that are relevant and significant when comparing these experiences differ from the factors that are relevant and significant for comparisons of experiences that differ merely in degree.

The objection, however, states that this difference in kind not only holds between experiences at the opposite ends of the spectrum; rather, the difference arises somewhere between adjacent experiences in the spectrum. For these experiences, due to their difference in kind, View One is not applicable, so the transitivity of "better than" is not threatened. That is, somewhere in the spectrum, two adjacent pains are of such different characters that the difference in time is not enough to outweigh the difference in kind and consequently we should reject View One.

I am inclined toward the gist of such an objection. However, Temkin is not convinced by it. His reply takes the following form: $P_{1}$ clearly differs in kind from $P_{n}$, and the objection states that there is a break somewhere in the spectrum from $P_{1}$ to $P_{n}$ where this change takes place. Let us call the two adjacent experiences for which View One does not hold $P_{k-1}$ and $P_{k}$. Temkin then asks whether View Three applies to $P_{k-1}$ and $P_{k}$ :

For our opponent's argument to work, he needs the difference between $P_{k}$ and $P_{k-1}$ 's pain to be akin to the difference between the pain of intense torture and the pain of a mosquito bite. If there were such a difference, then, indeed, we would agree that View Three applied to the two "nearby" pains $P_{k}$ and $P_{k-1}$; correspondingly, we would reject View One, and the threat to the transitivity of "better than" would evaporate.

(Temkin 2012: 272)

This response is misdirected, as Temkin focuses on View Three rather than View One. We should not assume that these views are mutually exclusive. The real issue is whether View One applies or not. The relevant question is thus whether the difference in kind is enough for View One not to apply. Temkin's view of this is that the difference is not enough. According to him, even though $P_{k-1}$ and $P_{k}$ may differ in kind, they are clearly very similar. For example, $P_{k-1}$ is more similar to $P_{k}$ than to $P_{1}$, and $P_{k}$ is more similar to $P_{k-1}$ than to $P_{n}$. Given that they are so very similar, we should expect View One to hold between them, according to Temkin.

Surprisingly, with this response, Temkin acknowledges that the experiences may indeed differ in kind. According to Temkin, however, the focus on different kinds is a red herring; instead, we should focus on the similarity of the adjacent experiences. He writes, 
In sum, though it may have helped illuminate how it could be the case that different factors could be relevant and significant for comparing different alternatives, the issue of different kinds is a red herring, and I ought not to have put some of my earlier discussions in those terms. The point is simply that when the difference between two pains is "sufficiently" great, View Three is appropriate for comparing them, whereas when the difference between two pains is "sufficiently" small, View One is appropriate.

(Temkin 2012: 274)

This, however, does not amount to a rebuttal of the claim that differences in kind constitute a counterexample to View One. Rather, Temkin seems to dig in his heels and once again claim that since $P_{k-1}$ and $P_{k}$ are next to each other in the spectrum, View One must be applicable, thus disregarding the claim that if $P_{k-1}$ differs in kind from $P_{k}$, then View One might not be applicable. The fact that $P_{k-1}$ and $P_{k}$ are adjacent does not rule out the possibility that they differ in kind. It is well-known that small changes can trigger larger changes, such as the proverbial straw that broke the camel's back. Small nonevaluative changes that trigger big changes in value are no less familiar. Adding a pinch of cilantro can, according to some, ruin an otherwise tasty dinner.

What one might find implausible in my suggestion, however, is that there is an abrupt change in the spectrum. One might find it implausible that along a fine-grained pain spectrum there exists an abrupt change in kind between two points. It may seem odd that there is such a sharp break in the spectrum (see Temkin 2012: 275).

Let us grant that a sharp break of this kind seems implausible. It could, however, still be that there is a break but that this break is not sharp. This is a familiar idea. A single grain of sand does not constitute a heap, but if we keep on adding grains of sand, we will eventually end up with a heap. There is, however, no exact lower limit on the number of grains of sand that is needed to rightly call the collection of grains "a heap." For some number of grains, it is indeterminate whether it is a heap or not. Similarly, there could be a zone in which it is indeterminate whether View One applies to the adjacent outcomes. ${ }^{8}$ It may, for example, be indeterminate whether the difference in kind is to be found between $P_{k}$ and $P_{k-1}, P_{k+1}$ and $P_{k}$, or $P_{k+2}$ and $P_{k+1}$. This means that although we cannot determinately judge for which outcomes in the spectrum View One does not apply, it will not be true for all adjacent experiences that View One will apply, since for $P_{k-1}$ to $P_{k+2}$ it will be indeterminate. This should make the claim more plausible.

Temkin (2012: 534-538) discusses a similar idea presented by Christopher Knapp (2007). Knapp argues that trade-offs between intensity and time seem to be desirable only when we are dealing with quantitative differences and not when it comes to qualitative differences. If 


\section{Henrik Andersson}

we have a spectrum ranging from very intense pain (600) to very mild pain (1), there is a difference in quality between the pains at the different ends of the spectrum, while two pains at the same end of the spectrum will only differ in quantity. For pains in the middle of the spectrum (300 and 301, for example), it is indeterminate whether they are very intense or very mild. ${ }^{9}$ And since it is indeterminate for two adjacent points in the middle of the spectrum whether there is a difference in quality, then it is indeterminate whether one of these outcomes that lasts for a certain time is better than its adjacent outcome that lasts much longer. Consequently, it is neither true nor false that View One holds and thus we have a break in the chain. ${ }^{10}$ However, Temkin is not much impressed by this objection:

We must clearly reject its key premise that a pain's being a borderline case of a qualitative distinction guarantees that it would be indeterminate whether or not View One or View Three applied for any comparisons involving that pain. This is simply not so. After all, even if one grants that pain 301 is both indeterminately very intense and indeterminately very mild, and so a borderline case of qualitative distinction, it doesn't follow that a trade-off between one year of pain 301 and five years of pain 301 would involve a borderline case where a qualitative difference was at stake! Since there is no difference between the intensity of pain in the two cases, only a difference in duration, it cannot be indeterminate whether a qualitative difference is at stake.

Admittedly, I find this reply somewhat unclear. It may be that he has shown that Knapp's "key premise" must be rejected, but this does not counter the more general reply that I have presented here.

However, Temkin continues his response to the line of reasoning I have presented here and provides a more general answer. According to him, it might very well be that it is indeterminate whether pains 300 and 301 are very intense or very mild, but it is determinate that the difference between them is small, and View One is appropriate to apply when we compare pains with a small difference between them in intensity.

Once again, Temkin seems to dig his heels in and claim that the two experiences are sufficiently similar, and by doing so he is neglecting the possible consequence of the difference in kind that holds between the experiences. His reply consequently seems to misconstrue the original objection. It was argued that - for the same reason that View One does not apply to experiences at the opposite ends of the spectrum - if adjacent experiences differ in kind, then View One is not applicable to these adjacent experiences. That is, if there is a difference in kind, then different criteria may be relevant to the comparison, blocking the chain of betterness relations. It may be indeterminate where this difference in kind lies 
on the spectrum, but it does, however, exist. For this reason, View One should be rejected. View One is supposed to be applicable to any two pains such that one is slightly less intense than the other. We now know that this is false since for some such pains there is a difference in kind, and for these pains, View One is not applicable.

By rejecting View One, Temkin's "most powerful” counterexample to the transitivity of "better than" fails, so there is no need to reject the view that "better than" is not a transitive relation.

\section{The Spectrum Argument and Value Superiority}

Let me try to make the same point but in a somewhat different manner. I take the issue at stake to be whether we should reject View One or View Four. Temkin has shown that if we accept the very reasonable Views Two and Three, Views One and Four will be incompatible. If we are to reject one of these views, we must have an explanation as to why the view might not hold. Temkin has given explanations as to why View Four might not always hold by arguing that the assessment of relative goodness is sometimes essentially comparative. However, accepting this as an explanation requires a revision of our conception of the nature of value: it would require that we "rethink the good." Moreover, View Four is fundamental to our conception of value, so if an explanation can be given as to why View One does not hold for all adjacent experiences in the spectrum, then this casts doubt upon Temkin's conclusion. I gave such an explanation above, so now we have reasons to doubt Temkin's conclusion.

Here I will give a somewhat more technical explanation as to why, given Views Two, Three, and Four, View One fails to apply. I will show that for two adjacent experiences in the spectrum, one will be weakly inferior to the other, which supports the claim that they differ in kind, and consequently, View One cannot hold for these two experiences. Thus, the chain of experiences allegedly connected in the manner described by View One is broken. So, by assuming transitivity, an explanation as to why View One fails to hold can be given. That is, from the knowledge we have of the structure of value, an explanation can be given as to why we should reject View One.

Interestingly, the argument I will present is similar to the view that Mozaffar Quizilbash, in this volume, attributes to James Griffin. Griffin refers to "discontinuity" and vagueness to show how the spectrum argument can be dismantled. I will not, however, use the term "discontinuity," but prefer the terms "superiority" and "inferiority." My argument will be based on research by Gustaf Arrhenius and Wlodek Rabinowicz (2015) regarding superiority in value. ${ }^{11}$ According to them, there are two kinds of superiority:

Strongly superior: An object $e$ is strongly superior to an object $e$ ' if and only if $e$ is better than any number of $e^{\prime}$-objects. 


\section{Henrik Andersson}

Weakly superior: An object $e$ is weakly superior to an object $e$ ' if and only if for some number $m, m e$-objects are better than any number of $e^{\text {'-objects. }}{ }^{12}$

(Arrhenius and Rabinowicz 2015: 232)

In their writings on the topic, Arrhenius and Rabinowicz (2015) have made the following interesting discovery:

Observation 5: Suppose that "is at least as good as" is a complete and transitive relation on the domain. Then, in any finite sequence of objects in which the first element is weakly superior to the last element, there exists at least one element that is weakly superior to its immediate successor. ${ }^{13}$

(Arrhenius and Rabinowicz 2015: 237)

Arrhenius and Rabinowicz's discussions are framed in terms of superiority; however, for the current discussion, it is more fitting to talk about strong and weak inferiority: ${ }^{14}$

Strongly inferior: An object $e$ is strongly inferior to an object $e$ ' if and only if $e$ is worse than any number of $e$-objects.

Weakly inferior: An object $e$ is weakly inferior to an object $e$ ' if and only if for some number $m, m e$-objects are worse than any number of $e$-objects.

We can now see that it is plausible that excruciating torture may be weakly inferior to the mild discomfort of a mosquito bite: two years of torture would always be worse than the discomfort of a mosquito bite, no matter how long the mosquito bite persisted.

I also take it that the results arrived at by Arrhenius and Rabinowicz are true for weak inferiority. The mirror image of the proof for Observation 5 should provide us with the proof for Observation 5'.

Observation 5': Suppose that "is at least as good as" is a complete and transitive relation on the domain. Then, in any finite sequence of objects in which the first element is weakly inferior to the last element, there exists at least one element that is weakly inferior to its immediate successor.

If we accept that experiences at the opposite ends of the spectrum are related by weak inferiority, which seems to fit well with Temkin's characterization of their relation, then it follows that somewhere along the spectrum, one experience will be weakly inferior to the next. This surprising result can be accounted for by the fact that there is a difference in kind between two adjacent items in the spectrum. ${ }^{15}$ Furthermore, it can be indeterminate where this change in kind takes place. ${ }^{16}$

Arrhenius and Rabinowicz's findings on superiority thus strengthen the support for the claim I made in the first part, i.e., that there is a 
difference in kind between two adjacent experiences. It has been shown that two adjacent experiences in the spectrum are related by a weak inferiority relation but it has not been shown that the chain of "better than" relations is broken. Even if $e_{k}$ is weakly inferior to $e_{k+1}$, it does not follow that $e_{k}$ is worse than $e_{k+1}$. The existence of weak inferiority in the spectrum should make us doubt View One, but the view has yet not been ruled out on formal grounds.

If Temkin's argument is to work, View One must apply to all adjacent experiences in the spectrum. View One claims that for any pain $e_{i}$, a slightly less intense experience that is twice (or three or five times) as long is worse. From this, it follows that for any two items in the spectrum, $e_{k}$ and $e_{k+1}, e_{k}$ is better than $e_{k+1}$. According to the definition of weak inferiority, for some number $m, m e_{k}$ objects are worse than any number of $e_{k+1}$ objects; $e_{k}$ may be better than $e_{k+1}$, and yet $m e_{k}$ may be worse than any number of $e_{k+1}$.

However, with one very reasonable assumption, we reach a contradiction. According to Temkin, $e_{k}$ is very similar to $e_{k+1}$, and if that is the case, it seems reasonable that if we prolong both experiences so that they become, for example, twice as long, then clearly $e_{k}$ would still be better than $e_{k+1}$. If 10 years of intense headache is better than 20 years of a slightly less intense headache, then clearly 20 years of intense headache must be better than 40 years of slightly less intense headache. Or, more generally, if $e_{k}$ is better than $e_{k+1}$, then $k e_{k}$ must be better than $k e_{k+1}$. This proportionality assumption entails that $m e_{k}$ is better than $m e_{k+1}$, which contradicts the result arrived at through the definition of weak inferiority: $m e_{k}$ objects are worse than any number of $e_{k+1}$ objects. This contradiction can be avoided by rejecting View One. ${ }^{17}$

The generality of the proportionality assumption can be questioned. It is doubtful if it is true for all contexts, and it may have boundaries such that it is not true for all values of $k$. For example, if $e_{k}$ and $e_{k+1}$ are two pleasurable experiences, it is possible that one has a diminishing marginal value while the other does not or that both have a diminishing marginal value but to different degrees. If that is the case, it is possible that $e_{k}$ is better than $e_{k+1}$ yet $k e_{k}$ is worse than $k e_{k+1}{ }^{18}$ The validity of the proportionality assumption can also be questioned when applied to extremely short experiences. One second of extreme pain may be better than a day of moderate pain, but 100 seconds of extreme pain may not be better than 100 days of moderate pain. ${ }^{19}$

However, for this context, i.e., for spectra of the kind discussed by Temkin, the proportionality assumption is plausible. We prefer the more intense experience to the less intense just because the latter is a longer experience - it is a trade-off we want to make. In this context, when $k$ is larger than 1 , the less intense experience becomes even proportionally longer than the more intense experience. This seems to be a trade-off that is in our favor. In fact, the consideration that speaks in favor of 


\section{Henrik Andersson}

the proportionality assumption seems similar to what speaks in favor of View One. According to View One, a negative experience will be better than a slightly less intense but much longer experience, so proportionally extending the experience should lead to the same result.

In other words, if one sets out to argue that the proportionality assumption is mistaken in this specific context, one will also struggle to defend View One. The intuitions that support the proportionality assumption in the context of Temkin's spectrum argument are hard to rebut since these concern views of trade-offs that seem central to the spectrum argument.

\section{Why We Should Accept Indeterminacy}

I have argued that Temkin's most convincing formulation of a spectrum argument does not lead to the conclusion that we should reject the transitive property of "better than." First, I argued that it is possible that somewhere in the spectrum there is a difference in kind between two adjacent experiences, and thus View One is not applicable between these experiences. Temkin's reply to this line of reasoning is not convincing, especially if one takes into consideration that it can be indeterminate where in the spectrum this change takes place.

Second, I explained why we should reject View One. It is not only that it is incompatible with the other three views; it was also shown that our knowledge of the structure of value relations provides a neat explanation as to how spectrum arguments can be blocked. Central to this explanation was the concept of weak inferiority. It could, of course, be objected that the possibility that one experience is weakly inferior to its adjacent experience is just as counterintuitive as the possibility that "better than" is not transitive. The explanation I have given is consequently not to be preferred to the explanation proposed by Temkin. Furthermore, the counterintuitive result may serve as another reason to reject View Four, since it is only if we accept transitivity that we can arrive at the result that one experience is weakly inferior to its adjacent experience. However, one must remember that there is no clear-cut boundary between experiences that differ in kind. It may be indeterminate where this change takes place since the borderline can be vague. Acknowledging the role of indeterminacy should thus make this strategy more appealing. Furthermore, the result is only counterintuitive if we believe that weak inferiority implies that the two experiences must differ greatly in value. This assumption might be premature, however, as Arrhenius and Rabinowicz (2015: 536) note: "contrary to appearances, $e$ might still be weakly superior to $e^{\prime}$, even though it is better only by a small margin." That is, even if they do not differ greatly in value, there can be a difference in kind between the two adjacent experiences. Given such a difference, one can doubt whether View One holds for these two experiences: if one experience can 
be weakly inferior to its adjacent experience, then it might be that these experiences are so different that View One does not apply.

However, an interesting result from Toby Handfield and Wlodek Rabinowicz (2018) may make my suggestion seem less plausible. They have argued that the indeterminacy needs to be of a certain kind to block the result of spectrum arguments, i.e., it needs to be radical. This is how they characterize radical indeterminacy that blocks a spectrum argument involving harms:

Two harm types admit radical indeterminacy if and only if, for at least one of them, $\mathrm{H}$, for all quantities $\mathrm{K}^{\prime}$ of $\mathrm{H}$ at least as large as some threshold $\mathrm{K}$, it is indeterminate for every sufficiently large quantity of the other type, $\mathrm{H}^{\prime}$, whether $\mathrm{K}^{\prime} \mathrm{H}$ is worse than this quantity of $\mathrm{H}^{\prime}$.

(Handfield and Rabinowicz 2018: 2384)

While weak inferiority and indeterminacy are sufficient to explain why a specific spectrum argument will fail, radical indeterminacy is needed to block the possibility of constructing a new spectrum argument. That is, even if there is a break in the spectrum and it is indeterminate where this takes place, it could be possible to construct a new spectrum by multiplying the number of items in the spectrum so that the indeterminacy disappears. With a sufficient number of items, it should become determinate what value relation obtains between the items, so a new spectrum argument can be made. The indeterminacy must consequently be radical to avoid this possibility. What makes their findings relevant here is that they argue that radical indeterminacy is hard to accept, making the strategy I have proposed less appealing. Handfield and Rabinowicz (2018) present two reasons meant to support the claim that it is hard to accept radical indeterminacy. It is, however, not clear that these considerations put in doubt the existence of radical indeterminacy. First, they discuss whether "indeterminacy that arises from a vague threshold in one relevant dimension must eventually be overwhelmed by a large enough difference in a second relevant dimension" (Handfield and Rabinowicz 2018: 2384). If that were the case, then appealing to indeterminacy to block spectrum arguments would be a dead end. However, they admit that no research suggests that indeterminacy must behave in this manner and that they lack robust intuitions on this matter. This reason should thus not be attributed too much weight. The second reason as to why radical indeterminacy is expressed as follows:

[R]adical indeterminacy arguably does not do very much to directly address the paradoxical features of spectra that exhibit radical inferiority between the first harm type and the last. As we have seen, if we assume that the betterness ranking across such a spectrum is complete, then there are adjacent, qualitatively very similar harm types in 
the spectrum such that one is radically inferior to the other. This is still counterintuitive, whether or not it is indeterminate where this point occurs. If it indeed is indeterminate, then - as we have seen radical indeterminacy will have to obtain between some adjacent types in the spectrum. Alternatively, if there is no radical inferiority between any two adjacent types, then we know that the only way to avoid violating transitivity is if at some point in the spectrum there is radical incommensurability between two adjacent types. Again, this remains surprising, even if it is indeterminate where this point occurs.

(Handfield and Rabinowicz 2018: 2384)

This is indeed true, but it is not a reason to reject radical indeterminacy; rather, it is a statement concerning the difficulties of making sense of spectrum arguments. It is true that spectrum arguments force us to accept some counterintuitive claims. In this chapter, I have argued that if we find intransitivity too hard to accept, we should, in the terminology of Handfield and Rabinowicz, accept radical indeterminacy.

Furthermore, Rabinowicz (2021) has recently argued that radicality of this kind may not, after all, be too difficult to explain. If we accept his fitting-attitudes analysis of value relations it is, for example, possible to account for some forms of such radicality. ${ }^{20}$

\section{Spectrum Arguments and Non-conventional Value Relations}

Throughout this chapter, I have assumed that the "at least as good" relation fully exhausts the domain of value relations. As evident in this volume, this assumption can and has been questioned. Several arguments have been presented to show that there might be additional sui generis value relations (e.g., Chang 2002; Rabinowicz 2008). Notions such as "parity," "incomparability," "imprecision," and "rough comparability" have all been invoked to attempt to dismantle the tension in spectrum arguments. For example, it has been argued that there is no need to assume that two adjacent experiences either are related by an at least as good as relation or are incomparable, as they might just as well be on a par. This relation between two adjacent experiences would block the spectrum argument (see Qizilbash 2007; Chang 2016), though it is not a promising strategy. First, much doubt has been expressed as to whether such a relation is plausible. Second, as Anders Herlitz (2020b) has shown, Handfield and Rabinowicz's arguments applied explicitly to parity allow us to conclude that radical parity must be confused since it is in tension with how parity is usually characterized. ${ }^{21}$

The approach taken here could be understood as part of a larger endeavor. My argument could be understood as part of the attempt to fully account for all value relations by referring to the concept of "at 
least as good." Several arguments put pressure on the assumption that "at least as good" fully exhausts the domain of value relations. These arguments, however, face the challenge of explaining why phenomena such as "parity," "incomparability," "imprecision," and "rough comparability" cannot be accounted for in terms of the vagueness of "at least as good." I and others have argued that this more conservative approach is just as successful in correctly depicting the axiological landscape (e.g., Broome 1997; Andersson 2017; Elson 2017). It has now been shown that this more conservative approach can also help explain why "better than" is a transitive relation, so there is no need to introduce any further value relations to arrive at this result.

To give an example, my objection to Temkin's argument resembles Derek Parfit's view of a similar matter. When discussing the related topic of the Repugnant Conclusion, Parfit (1984: 110) argues that the notion of imprecision allows us to avoid the conclusion that

[c]ompared with the existence of many people who would all have some very high quality of life, there is some much larger number of people whose existence would be better, even though these people would all have lives that were barely worth living.

While he refers to something similar to weak inferiority to avoid this conclusion, he also needs to introduce the notion of "imprecision." This approach could perhaps successfully show why we need not accept Temkin's conclusion that "better than" is not transitive. However, my account has the advantage of only appealing to the familiar notions of vagueness and indeterminacy; there is no need to introduce a further mysterious notion.

\section{Conclusion}

This chapter's result is important because it clarifies how Temkin's four views are incompatible. For those who find it hard to reject Views Two, Three, and Four, an explanation has now been given as to why View One ought to be rejected: There is a difference in kind between experiences at the opposite ends of the spectrum, and this difference arises between two adjacent experiences. Since there is such a difference in kind, View One is not applicable and the chain of betterness relations does not hold throughout the spectrum. The findings regarding the structure of value arrived at by Arrhenius and Rabinowicz neatly support the claim that if we accept Views Two, Three, and Four, we must reject View One. One experience will be weakly inferior to its adjacent experience, so View One is not applicable. This weak inferiority can be accounted for by the fact that the experiences are of a different kind. Furthermore, it can be indeterminate where this change takes place. 


\section{Henrik Andersson}

When we face incompatible claims, we need an explanation as to which claim we should reject. Temkin has explained why the transitivity of "better than" might not always hold. Now we also have an explanation as to why View One might not always hold.

\section{Notes}

1 I owe much gratitude to many for helping me develop the ideas presented here. A first version of this chapter that included the main argument was written in 2017, and several versions have since been circulated and presented on various occasions. The feedback I received from Erik Carlson during my $\mathrm{PhD}$ defense inspired me to write the chapter in the first place. Since then I have received helpful comments from Mattias Gunnemyr, Anders Herlitz, Jakob Werkmäster, Wlodek Rabinowicz, the audience at the Hard Cases and Rational Choice workshop in Bern in November 2017, and the audience at the Higher Seminar in Practical Philosophy in Lund at which this chapter was presented.

2 Throughout most of this chapter, I will assume that there are only three value comparatives: "better than," "worse than," and "equally as good as." If there are other value comparatives beyond these three, then it is, of course, possible that these additional comparatives are not transitive. I will return to the importance of this assumption.

3 He discusses the very specific value relation the "all things considered better than in a wide reason-implying sense.” Temkin (2012: 13) describes the relation in the following way: "Roughly, on this use, outcome $A$ is better than outcome $B$, all things considered, if one would have more reason to prefer $A$ to be realized than $B$, from an impartial perspective."

4 An early formulation of this can be found in Temkin (1996); the argument is, however, inspired by an argument by Stuart Rachels (1998).

5 John Broome (1997) makes perhaps the most important contribution to the attempt to provide an account of the normative landscape without referring to non-conventional value relations; see also Constantinescu (2012), Andersson (2017), Elson (2017), and Broome (2021).

6 All four views are grounded in intuitions that are common in axiological theorizing. If we are to reject one of them, then this will have consequences for theories that encompass them. The practical problems of rejecting any of the views is because they are relevant when discussing trade-offs. As Temkin (2012: 23) acknowledges, practical decisions often involve trade-offs. Tradeoffs are therefore central for many of our everyday decisions, as well as for life-changing decisions. The philosophical literature often connects these discussions to population axiology and the distribution of benefits, i.e., discussions that clearly involve trade-offs, but they are also relevant to more mundane situations since these also often involve trade-offs. The cost of rejecting View Four can also be accounted for by referring to rational choice theory. Value relations are often believed to guide us to rational choice among alternatives. However, if the betterness relation can be cyclical, then we will not be guided toward the best alternative. For a more nuanced view of the practical implications of rejecting View Four, see Herlitz (2020a).

7 There could be several such breaks along the spectrum but, for the objection to hold, one suffices.

8 In fact, there could be several such zones, but in line with the discussion in note 7 , one zone is all that is needed for the objection to hold. 
9 I take this to be a simplified version of Temkin's description of the spectrum. It is more plausible that the spectrum consists of several categories and that these categories have indeterminate boundaries. Consequently, the pains in the middle are neither very intense nor very mild. However, the important point is correct even in the simplified description: it can be indeterminate where the difference in kind is found.

10 Not only is it indeterminate whether View One applies and an experience is better than its adjacent much longer experience, but it is also indeterminate whether the same experience is worse than the much longer experience.

11 The idea of superiority in value be traced back to at least John Stuart Mill (1863).

12 James Griffin (1986) had a similar distinction in mind.

13 Very roughly, the proof of the observation starts by proving the following: "Lemma 1: Suppose that 'is at least as good as' is a weak order, that is, a complete and transitive relation on the domain. For any objects $e, e^{\prime}$, and $e^{\prime \prime}$, if $e$ is weakly superior to $e^{\prime \prime}, e$ is weakly superior to $e^{\prime}$ or $e^{\prime}$ is weakly superior to $e^{\prime \prime}$ " (Arrhenius and Rabinowicz 2015: 241). Observation 5, of course, entails Lemma 1 and that Lemma 1 entails Observation 5 can also be shown. Lemma 1 is then proven by assuming that (i) $e$ is weakly superior to $e^{\prime \prime}$, but (ii) $e$ is not weakly superior to $e^{\prime}$. To establish Lemma 1, we need to show that $e^{\prime}$ is weakly superior to $e^{\prime \prime}$. From (i) we know that there must exist some number $m$ for which (1) $m e$-objects are better than any number of $e^{\prime \prime}$-objects. From (ii) we can infer that there exists some number $m^{\prime}$ for which "(2) $m$ e-objects are not better than $m^{\prime} e^{\prime}$-objects. But then, given that 'is at least as-good as' is a complete relation, (2) implies that (3) $m^{\prime} e^{\prime}$-objects are at least as good as $m$ $e$-objects. By the transitivity of 'is at least as good as', if one object is at least as good as another, which is better than some third object, then the first object is better than the third. Consequently, (3) and (1) imply that (4) $m^{\prime} e^{\prime}$-objects are better than any number of $e^{\prime \prime}$-objects. (4) implies that $e^{\prime}$ is weakly superior to $e^{\prime \prime} "$ (Arrhenius and Rabinowicz 2015: 242).

14 While the idea of superiority easily translates to inferiority, it is also possible to consider similar structures for "equally as good" and non-conventional value relations, such as "parity." For more on this matter, see Henrik Andersson and Andrés Garcia (n.d.).

15 This view seems to be endorsed by James Griffin (1986: 338-340). For a helpful discussion of Griffin's view, see Mozaffar Qizilbash (2005, 2021). This view also shares some features with Derek Parfit's (1984) attempt to avoid the Repugnant Conclusion. For more on the similarities and differences, see the following under "Spectrum Arguments and Non-Conventional Value Relations” and Qizilbash (2021).

16 As Qizilbash has argued, if one finds the suggestion of one experience being inferior to its adjacent experience to be implausible, one should take into account that the boundary between these experiences may be vague. Qizilbash (2005) does not use the same terms but has framed his discussion in terms of Griffin's “discontinuity.”

17 It could, of course, also be avoided by rejecting the claim that the first experience is weakly inferior to the last. However, Temkin seems to endorse this inferiority claim, which, after all, is almost exactly what his View Three states.

18 This possibility was suggested to me by Wlodek Rabinowicz.

19 I owe this example to Anders Herlitz.

20 Rabinowicz (2021) provides an explanation of radical parity and not radical indeterminacy. Roughly, radical parity can be accounted for in terms of a class of permissible preference orderings that differ from one another as to where 


\section{Henrik Andersson}

the break in the spectrum is located. The fact that Rabinowicz's account shares structural features with the so-called supervaluationistic account of vagueness makes the possibility of explaining the occurrence of radical indeterminacy within this framework promising. For more on the concept of "parity", see the following.

21 More specifically, it is in tension with the so-called chaining argument that is an essential part of the argument for a fourth possible value relation. See also Rabinowicz (2021) for an attempt to explain radical parity.

\section{References}

Andersson, H. (2017), How It All Relates: Exploring the Space of Value Comparisons (Media-Tryck).

Andersson, H., and Garcia, A. G. (n.d.), 'Millian Value Relations Generalised and Interpreted'.

Arrhenius, G., and Rabinowicz, W. (2015), 'Value Superiority', in I. Hirose and J. Olson (eds.), The Oxford Handbook of Value Theory (Oxford University Press).

Broome, J. (1997), 'Is Incommensurability Vagueness?', in R. Chang (ed.), Incommepnsurability, Incomparability, and Practical Reason (Harvard University Press), 67-89.

Broome, J. (2021), 'Incommensurateness Is Vagueness', in H. Andersson and A. Herlitz (eds.), Value Incommensurability: Ethics, Risk, and Decision-Making (Routledge).

Chang, R. (2002), 'The Possibility of Parity', Ethics 112/4: 659-688.

Chang, R. (2016), 'Parity, Imprecise Comparability and the Repugnant Conclusion', Theoria 82/2: 182-214.

Constantinescu, C. (2012), 'Value Incomparability and Indeterminacy', Ethical Theory and Moral Practice 15/1: 57-70.

Elson, L. (2017), 'Incommensurability as Vagueness: A Burden-Shifting Argument', Theoria 83/4: 341-363.

Griffin, J. (1986), Well-Being: Its Meaning, Measurement and Moral Importance (Clarendon Press).

Handfield, T., and Rabinowicz, W. (2018), 'Incommensurability and Vagueness in Spectrum Arguments: Options for Saving Transitivity of Betterness', Philosophical Studies 175: 2373-2387.

Herlitz, A. (2020a), 'Non-Transitive Better Than Relations and Rational Choice', Philosophia 48/1: 179-189.

Herlitz, A. (2020b), 'Spectrum Arguments, Parity and Persistency', Theoria 86/4: 463-481.

Knapp, C. (2007), 'Trading Quality for Quantity', Journal of Philosophical Research 32/1: 211-233.

Mill, J. S. (1863), Utilitarianism.

Qizilbash, M. (2005), 'Transitivity and Vagueness', Economics and Philosophy 21/1: 109-131.

Qizilbash, M. (2007), 'The Mere Addition Paradox, Parity and Vagueness', Philosophy and Phenomenological Research, 75: 129-151.

Qizilbash, M. (2021), 'On "Incommensurability," "Discontinuity" and the Repugnant Conclusion: “Imprecise Equality” or Vagueness?', in H. Andersson 
and A. Herlitz (eds.), Value Incommensurability: Ethics, Risk, and DecisionMaking (Routledge).

Parfit, D. (1984), Reasons and Persons (Oxford University Press).

Parfit, D. (2016), 'Can We Avoid the Repugnant Conclusion?', Theoria 82/2: 110-127.

Rabinowicz, W. (2008), 'Value Relations', Theoria 74/1: 18-49.

Rabinowicz, W. (2021), 'Can Parfit's Appeal to Incommensurabilities in Value Block the Continuum Argument for the Repugnant Conclusion?', in J. McMahan, T. Campbell, J. Goodrich, and K. Ramakrishnan (eds.), Ethics and Existence: The Legacy of Derek Parfit (Oxford University Press).

Rachels, S. (1998), 'Counterexamples to the Transitivity of 'Better Than", Australasian Journal of Philosophy 76/1: 71-83.

Temkin, L. (1996), 'A Continuum Argument for Intransitivity', Philosophy \& Public Affairs 25/3: 175-210.

Temkin, L. (2012), Rethinking the Good: Moral Ideals and the Nature of Practical Reasoning (Oxford University Press). 